\title{
Discussion on the Construction and Application of Navigation Electronic Map Quality Inspection Sample Database
}

\author{
Jin Bai ${ }^{1}$, Jingnan $\mathrm{Gao}^{2, *}$, Wei Ma ${ }^{1}$, Qingqing Yan $^{1}$, Xiaodi Wang ${ }^{1}$, and Shuai Dong ${ }^{1}$ \\ ${ }^{1}$ National Quality Inspection and Testing Center for Surveying and Mapping Products, 100830 Beijing, China \\ ${ }^{2}$ SpaceWill Info. Co., Ltd, 100089 Beijing, China
}

\begin{abstract}
In order to improve the scientificity and efficiency of quality inspection of navigation electronic map, and save the cost of quality inspection, it is necessary to establish the national navigation electronic map quality inspection sample database. In this paper, the construction goal, data acquisition technology process, and database construction method of navigation electronic map quality inspection sample database are described in detail, and the construction content and technical indicators are discussed. The quality test is carried out by combining the in-house verification with the field comparison of the navigation electronic map quality test sample database, and the test results are analyzed and evaluated. The effective application of the sample database in the quality test and evaluation of the internet navigation electronic map in the pilot area is carried out. At the same time, the application of navigation electronic map quality inspection sample database in the research of urban fine management and solid waste recycling economy ecological park planning is prospected.
\end{abstract}

\section{Introduction}

With the development of communication, electronics, network technology, and the geographic information industry, map navigation products have been widely used in people's daily life [1-2]. The mobile terminal navigation system and vehicle navigation have become necessary tools for people to travel. As a form of a public map, Internet navigation electronic map has developed rapidly in recent years. According to incomplete statistics, there are more than 40000 Internet map service websites in China. Due to the differences in service fields, service modes, and methods, there are great differences in map basic data, map display, and service functions among various Internet navigation electronic map service websites. However, when users use electronic map navigation and positioning products of different manufacturers, they find that some products often have different degrees of quality problems. Due to a large amount of data, strong timeliness, and complex data structure of navigation electronic map, in the current navigation electronic map quality detection work, for each test sample area, we need to go to the scene for benchmark data collection and field testing. The establishment of national navigation electronic map quality inspection sample database has become a problem to be solved [3]. Therefore, it is very important to develop the construction and application of a sample database for navigation electronic map quality inspection, which is of great significance to improve the scientific and efficiency of the quality detection of navigation electronic map and promote the development of the geographic information industry.

\section{Data characteristics of navigation electronic map quality inspection sample database}

\subsection{Accuracy of point of interest retrieval}

As a typical application of navigation system, the accuracy of reaching the specified target can be used as an important parameter to evaluate the navigation system [4]. In common geographic information databases, point of interest (POI) usually represents geographic points and simple related information. But in the construction of navigation electronic map quality detection sample database, users need to retrieve the accurate entrance and exit of the specified target in the process of using the navigation system, so that the system can lay the foundation for users to plan the most accurate route in a short time. Therefore, in the construction of navigation electronic map detection sample database, especially for shopping malls, hospitals and other features with large area and multiple exits, it is necessary to show the routes of entrances and exits in all directions as much as possible.

\subsection{High data availability}

Vehicle navigation and mobile terminal navigation system are the two most widely used directions of navigation

\footnotetext{
${ }^{*}$ Corresponding author: 313219666@qq.com
} 
system at present. When users use the navigation system, whether they are using cars, sharing bicycles or other modes of transportation, they are most concerned about how to reach the destination with the optimal path in a short time. Navigation electronic map is an important basis of navigation system, and the current situation of data will directly affect the accuracy of navigation. The most frequent changes in the process of car driving are traffic restriction information and dynamic traffic information. The actual traffic guidance information and traffic condition information are the two indication conditions that must be followed in the process of car driving. Whether the information in the navigation system is consistent with the field information can be used to judge whether the driving instructions based on the navigation database are effective, Therefore, the current situation of traffic restriction information and dynamic traffic information can directly determine the efficiency of navigation electronic map detection process.

\subsection{Reasonable data topological relationship}

In the construction of navigation electronic map quality inspection sample database, the sample area data needs to express the geographic location, shape and spatial relationship of the road information and related element data in the target area. Taking road information detection as an example, the connectivity of road network is one of the concerns of navigation electronic map detection personnel, that is, the topological relationship between the arc segment and the node in the road layer, which contains the attributes of traffic capacity. In the process of building the database, when dealing with the topological relationship of Road intersection, especially in the multilevel Road intersection, overpass deck and other places, the topological relationship of the road should be fully reflected, and the combination of the topological relationship and the traffic capacity should be paid attention to at this time. The change of traffic capacity is usually at the node, and the decrease of traffic capacity may affect the traffic degree of topologically connected roads

\section{The implementation of sample database for navigation electronic map quality inspection}

\subsection{Technical process of sample data collection}

The sample database of navigation electronic map detection has been collected in 93 cities of 31 provinces, cities and autonomous regions nationwide, and 93 sample areas have been selected. The sample areas include municipalities directly under the central government, provincial capital cities, prefecture level cities and county-level cities. The regional distribution takes into account the East, South, West, North and central regions of China, and the degree of development takes into account the urban, urban-rural integration and rural areas. The sample area size is about $3 \mathrm{~km} \times 3 \mathrm{~km}$. According to the standard data collection scheme of navigation electronic map quality inspection sample database, realtime road network and its ancillary information data are collected in the sample area, including road network data, administrative division data, water system data of grade 6 and above, and basic data of place name data. Collect the road, background and notes of motor vehicles in the city; Collect the data of relevant points of interest in the city, and organize and process the data.

\subsection{Collection technical index}

\subsubsection{POI collection}

POI is the point of interest. Whether it is vehicle navigation or mobile terminal navigation, today's navigation systems on the market have their own POI database [5-6]. The number of POI in the navigation map, the accuracy of information and the speed of information update all seriously affect the use of a navigation system. The collection of POI should focus on the important information concerned by users, such as the accurate full name and relevant location information of interest points such as the location of the sample area government, local widely recognized landmark features, hotels, restaurants, shopping malls, transportation hubs, hospitals, gas stations, charging stations, schools, etc. In the process of field collection, it is necessary to collect the name information of POI on the basis of authoritative information, verify the location of POI or collect new POI point information on the spot, take photos on the spot, record the collection time of POI, and record the important attributes of POI, such as scenic spot level and hotel star level. It should be noted that the hotel star rating is subject to the official announcement of the tourism administration, and the Hotels with larger scale equivalent to 4 or 5 stars are classified as the secondary important category. In the process of in-house collation, the POI benchmark data is set up as a separate point layer. Based on the fields including number, name, collection and verification time, photo number and remarks, the POI benchmark data is sorted out, and the fields of classification code, importance and other important attributes are added.

\subsubsection{Road information collection}

The content of road information collection includes road network information, traffic restriction information, road guidance map information and so on.

The field work of road network information collection is mainly based on the image and the 1:10000 road data in the sample area to carry out on-site verification and mapping, and record the driving track in the whole process to complete the collection of road morphology and structure. In the process of field work, we should focus on collecting the level and code of high-level roads, and refer to the official data for confirmation and on-site verification before the survey. In the process of office work arrangement, it is necessary to establish the line layer of road information separately, specify the direction 
of road acquisition as the traffic flow direction, and draw all the road elements collected and verified by the field work in the survey area on the image according to the field work track information and 1:10000 road data. In the road information layer, key fields such as reference name, road code, level, one-way restriction, one-way restriction time domain, number of lanes and important one-way line are added.

In navigation electronic map data, structure information, one-way traffic information, and intersection traffic restriction information are combined to express traffic restriction information. It can be divided into important one-way lines and all intersection restriction information, among which important one-way lines, including roundabout, single lane road, often match with traffic restriction information. In the field work, 1:10000 data is used as the base map to collect and identify the single lane, note its time domain, road section range, flow direction and other information, and collect and number the photos related to the single lane information. The basic traffic restriction information includes no left, no right, no U-turn and no straight. In the office work, it is necessary to establish a point layer for traffic restriction information, and add key fields such as road level, restriction information description and vehicle type in the layer.

\subsubsection{Background and annotation information collection}

The background element information of navigation electronic map data involves administrative division, water system, railway and green space information, and the annotation information involves road name and number, public facilities name and other information. In the construction of navigation database, the background and annotation information need to refer to the basic 1:10000 data acquisition, field verification and photo recording, and the internal industry should build a separate layer and set up the number, name, acquisition / update time, photo number and other fields.

\section{Application test of navigation electronic map quality inspection sample database}

Organize the quality inspection practitioners to carry out the quality inspection test by combining the in-house verification and field comparison in the navigation electronic map quality inspection sample database as a group, and analyze and evaluate the test results. The evaluation methods and experimental results are as follows:

\subsection{Evaluation method}

The score of test results is calculated according to the proportion of errors and omissions of different levels, and the score is shown in the formula (1).

$$
S_{2}=100-\left[a_{1} *(12 / t)+a_{2} *(4 / t)+a_{3} *(1 / t)\right]
$$

In formula (1), $S_{2}$ is the score of mass element; $a_{1}$ is the number of serious errors; $a_{2}$ is the number of large errors; $a_{3}$ is the number of general errors; $t$ is the adjustment factor of deduction.

The comprehensive score of elements is calculated by weighted average according to the detection index, and the calculation method is shown in the formula (2).

$$
S_{I}=\sum_{i=1}^{\mathrm{n}}\left(S_{2 i} * p_{i}\right)
$$

In formula (2), $\mathrm{S}_{1}$ is the score of the corresponding mass element; $\mathrm{S}_{2 \mathrm{i}}$ is the score of the corresponding mass sub elements; $\mathrm{P}_{\mathrm{i}}$ is the weight of corresponding quality sub elements, which can be adjusted according to the emphasis of inspection; $n$ is the number of quality sub elements contained in the quality element. For a sample area, the formula for calculating the weighted score of all unit results is:

$$
S_{1}=\sum_{i=1}^{n}\left(S_{1 i}^{*} p_{i}\right)
$$

In formula (3), $S_{1}$ is the data quality score of a sample area; $S_{1 i}$ is the score of each mass element; $P_{i}$ is the weight of each quality element can be adjusted according to the emphasis of inspection; $\mathrm{n}$ is the number of quality elements contained in the unit achievement.

\subsection{Analysis of experimental results}

Based on the evaluation method of this paper, six influential Internet navigation electronic map service websites are selected to carry out the experiment. The sample area of the experiment is mainly in Prefecture and county-level cities. Six cities are randomly selected from the sample database of navigation electronic map quality detection. In each sample area, according to the complexity of road and poi, the area of not less than 4 square kilometers is cut as the sample area of the actual test.The data in the navigation electronic map quality inspection sample database obtained by field measurement is taken as the reference data of the sample area. Tile map sample data is obtained online and realtime by ordinary users through the official websites of various map service providers. In the experiment, the query function provided by the website is used to query the POI data one by one, and compare with the benchmark reference data to determine the integrity and consistency of the data. The integrity and correctness of the road data are verified by using the line planning function. The test results are shown in Figure 1.

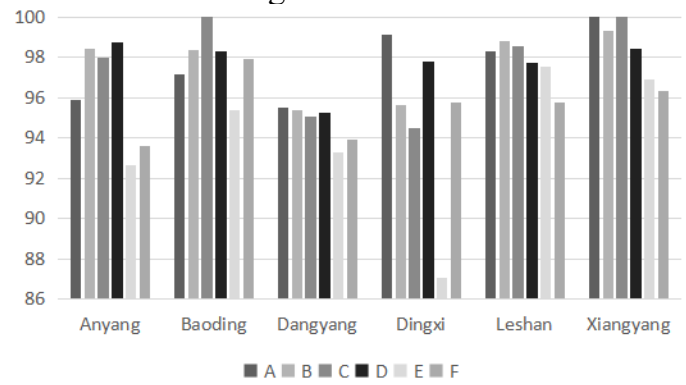

Figure 1. Score results of internet navigation electronic map service test sample area. 
The above test results show that the quality evaluation method based on the navigation electronic map quality inspection sample database can not only evaluate the quality of Internet navigation electronic map service well, but also reflect the fine differences in data and services of various Internet map service providers.

\section{Prospect of application scenarios}

\subsection{Application of sample database data in urban fine management}

Urban management is the coordinated control behavior of all the activities of the operation and development of the city. Fine management is a management concept and technology that originated from Japanese enterprises in the $1950 \mathrm{~s}$, which has been applied to all kinds of social organizations [7]. Urban fine management is to introduce the concept of fine management into urban management. In order to implement fine management in a city, it is necessary to refine the management of underground pipe networks, roads, bridges, buildings and other objects of underground, ground, and above ground space. With the passage of time, the city is constantly changing. The navigation electronic map quality inspection sample database data can more clearly and objectively display and mine the information of urban geospatial-related elements. The timely updated geographic information data can realize the regular observation of large urban areas, and help to grasp the panorama and historical changes of the city. It will greatly promote the application of geographic information data in all aspects of urban fine management. Using high timeliness geospatial information to carry urban management business information, truly restore urban road construction and other information, spatially and sequentially process urban management related business data, and carry it into the urban space-time environment based on geographic information data, so as to provide data support for urban meticulous management.

\subsection{Application of sample database data in the planning of solid waste recycling economy ecological park}

Industrial Park is an important entry point for the development of circular economy and sustainable development. [8-9]. By using the limited available site space and concentrating all kinds of municipal solid waste treatment facilities, the construction of a circular economy eco-industrial park for municipal solid waste treatment can not only reduce the resistance caused by scattered-site selection, but also improve the joint treatment and disposal level of various types of municipal solid waste, at the same time, it is also conducive to the construction of material, energy and water resources recycling cascade utilization system, which is an effective mode to solve the problem of "garbage siege" in large and medium-sized cities with the land shortage. Using the navigation electronic map quality inspection sample database to obtain the surrounding features information and spatial distribution of the park, to provide basic data support for the planning of the park land layout structure. Combined with the surrounding road planning, congestion information, the process characteristics of each project, and the material supply and demand relationship between projects, the optimal layout of all kinds of land and projects in the park is realized, it provides a scientific and effective plan for the planning and construction of the solid waste park.

\section{Summary}

As an important foundation of the navigation system, the quality of the navigation electronic map will directly affect the user's evaluation of the navigation system. It is the key to guaranteeing the quality of all kinds of electronic navigation system products to strictly control the quality of navigation electronic maps by adopting the industry technical standards and Technical Specifications issued by state organs. The quality inspection of navigation electronic map in some pilot areas has been confirmed. Through scientific and feasible methods, we can establish a multi-level navigation electronic map quality inspection sample database covering the whole country, and carry out the application of navigation electronic map quality inspection sample database, which can save the inspection cost on the basis of ensuring the authenticity and reliability of navigation electronic map data inspection results Improve inspection efficiency, and continuously improve the quality of navigation electronic map, Internet map service products, and navigation terminal products. At the same time, the establishment of a year-by-year updated navigation electronic map quality testing sample database can not only serve the quality control of Internet map quality testing. It can also be applied to the research of urban fine management, carbon emission monitoring and management, solid waste recycling economy ecological park planning, and so on. It provides a new idea for the above research.

\section{References}

1. X.C. Liu, J. Liu, D.L. Li, Bull Surv Map. 33, 5 (2012)

2. Y.H. Cai, W. Zhang, Journal of Navigation and Positioning. 10, 1 (2021)

3. H.Q. Yang, Bull Surv Map. 37, 11 (2014)

4. J. Zhang, N. Jiang, H. Zhou, Geomatics \& Spatial Information Technology. 55, 2 (2015)

5. P. Gao, A. H. Wu, Beijing Surv Map. 24, 1 (2013)

6. Q.Q. Yan, X. Ma, H. Li, Geomatics \& Spatial Information Technology. 169, 5 (2016)

7. L.Q. Guo, J.Y. Lin, W. Y. Wang, Urban Development Studies. 57, 1 (2012)

8. J.M. Hong, M. Li, Environment Engineering Design. 155, 1 (2020)

9. C.X. Hao, Natural Science. 175, 7 (2019)

10. X.M. Su, J.C. Tan, Y.Z. Chen, Beijing Surv Map. 126, 3 (2016) 